\title{
Expositions nationales et nation helvétique : la quête d'identité
}

\section{Pierre Centlivres}

\section{OpenEdition}

\section{Journals}

Édition électronique

URL : http://journals.openedition.org/ress/263

DOI : $10.4000 /$ ress.263

ISSN : 1663-4446

Éditeur

Librairie Droz

Édition imprimée

Date de publication : 1 août 2006

Pagination : 123-143

ISBN : 9-782-600-01108-2

ISSN : 0048-8046

\section{Référence électronique}

Pierre Centlivres, "Expositions nationales et nation helvétique : la quête d'identité », Revue européenne des sciences sociales [En ligne], XLIV-135 | 2006, mis en ligne le 13 octobre 2009, consulté le 02 mai 2019. URL : http://journals.openedition.org/ress/263 ; DOI : 10.4000/ress.263 
Pierre CENTLIVRES*

\section{EXPOSITIONS NATIONALES ET NATION HELVÉTIQUE: LA QUÊTE D'IDENTITÉ}

Pour Dominique Schnapper, qui possède une connaissance étendue, inhabituelle même, du système et de la culture politiques de la Suisse, notre pays apparaît comme un des exemples d'une «nation de volonté» (Willensnation) fondée davantage sur un projet politique partagé que sur une communauté culturelle ou que sur le mythe d'une origine unique. «La communauté des citoyens peut, dans son principe, être culturellement hétérogène, comme le montre l'exemple toujours évoqué de la Suisse, l'homogénéité culturelle des populations n'étant qu'un facteur favorisant la constitution d'une société politique.» ${ }^{1}$

Dans un autre chapitre de La communauté des citoyens, elle revient sur le multiculturalisme helvétique transcendé par le projet fédéral commun. «Les Suisses peuvent se vanter de ce que leur projet politique fasse coopérer en une entité politique unique des populations utilisant quatre langues, appartenant à deux grandes religions et à de nombreuses Eglises, à des entités politiques dont les traditions étaient diverses...» ${ }^{2}$

Pourtant, depuis les années quatre-vingts et quatre-vingt-dix, et même avant ${ }^{3}$, les médias et l'opinion font preuve de retenue, pour dire le moins, dans l'expression de la fierté helvétique. Le désenchantement, voire le désamour font suite à la prise de conscience d'un passé national récent médiocre et à la mise à mal de l'exemplarité de la Suisse ${ }^{4}$.

Certes, D. Schnapper ne méconnaît pas les faiblesses, ou les risques, inhérents à la nation-contrat: "Elle ferme la nation au passé, rompt les liens entre les individus créés par une identité, une culture, des traditions communes et ne peut manquer d'affaiblir à l'extrême le lien social jusqu'à risquer de le dissoudre.» ${ }^{5}$

Les faiblesses structurelles d'une Suisse qui n'est pas une «NationalitéEtat $»^{6}$, donc dont la base n'est pas ethnique, et qui «peut être considérée comme

\footnotetext{
* Professeur émérite de 1'Université de Neuchâtel et ancien directeur de l'Institut d'ethnologie; Pierre.Centlivres@unine.ch

1 D. Schnapper, La communauté des citoyens. Sur l'idée moderne de nation, Paris, Gallimard, 1994, p. 43-44.

2 Ibid., pp. 127-128.

3 Voir M. Imboden, Helvetisches Malaise, Zurich, Polisverlag, 1964.

4 P. Centlivres, «Le portrait introuvable: la Suisse des expositions nationales », Ethnologie française XXXII/2, 2002, pp. 311-320.

5 D. Schnapper, La France de l'intégration. Sociologie de la nation en 1990. Paris, Gallimard, 1991 , p. 50.

6 A. Van Gennep, Traité comparatif des nationalités. (Tome 1: Les éléments extérieurs de la nationalité), Paris, Payot, 1922, p. 26.
} 
la réunion artificielle de trois marches ${ }^{7}$, sont des thèmes récurrents aujourd'hui.

Je me propose ci-dessous d'aborder les thèmes de l'identité et de la cohésion de la Suisse «par la bande», à partir d'images et de manifestations emblématiques. Les Expositions nationales figurent explicitement et implicitement parmi ces faits sociaux et symboliques qui représentent les aspirations, les mythes et les hantises d'une nation suisse en devenir depuis la Constitution de 1848. Par-delà leur rôle de foires industrielles et agricoles, elles donnent à voir ce que les classes dominantes ont appelé, de la fin du XIX ${ }^{\mathrm{e}}$ siècle au milieu du XX $\mathrm{XX}^{\mathrm{e}}$ siècle, le Sonderfall Schweiz, le cas particulier de la Suisse comme exception politique et modèle au cœur de l'Europe, mais aussi comme fondement mythique de l'identité helvétique ${ }^{8}$.

La Suisse a mis sur pied, de Zurich en 1883 à la région des Trois Lacs de Neuchâtel, de Bienne et de Morat en 2002 (Expo.02), six Expositions nationales. Jusqu'en 1939 au moins et avec une approche plus critique ensuite, ces grandes manifestations ont eu pour ambition d'être des vitrines pour les produits de l'industrie et des arts, d'exalter ainsi les progrès de la Suisse industrielle et moderne avec l'aide d'une mise en scène grandiose et l'appui des institutions de l'Etat'. Elles visaient aussi à rappeler aux Suisses leur unité difficilement constituée, par-delà le cloisonnement géographique et l'hétérogénéité culturelle, et à leur montrer «le visage aimé de la Patrie», au travers d'une success story.

Aussi, les études historiques et sociologiques qui lui sont consacrées font une large place, à côté des enjeux économiques, aux thématiques par lesquelles s'exprime le «national» de ces expositions, soit aux thèmes du «reflet» de la réalité helvétique $^{10}$, de l'identité $^{11}$, du modèle réduit ${ }^{12}$, ainsi qu'à ceux de la cohésion et du consensus. Elles analysent en particulier l'exaltation des valeurs mythiques d'une Suisse archétypale, alpestre et paysanne, chère aux milieux conservateurs, mais pas à eux seulement. (v. par exemple: Expos.ch, Berne 2000, ou Quand la Suisse s' expose, Neuchâtel 2002).

L'analyse du contexte socio-économique dans lequel s'inscrivent ces manifestations n'est pas mon propos ici. Dans les pages qui suivent, j'aimerais reprendre certains de ces thèmes, leurs variations, leurs transformations au cours des expositions nationales successives. Je tenterai également de présenter certains des moyens utilisés pour combler symboliquement le déficit helvétique en substance identitaire et masquer la réalité conflictuelle du moment. Enfin, je chercherai à montrer la rupture radicale que représente la plus récente d'entre elles (la dernière?), celle de 2002, Expo.02.

${ }^{7}$ Ibid., p. 159.

8 A. Reszler, Mythes et identité de la Suisse. Genève, Georg, 1986.

${ }^{9}$ P. Centlivres, «Le portrait introuvable: la Suisse des expositions nationales», op. cit., p. 312.

${ }^{10}$ Les Suisses dans le miroir. Les expositions nationales suisses. De Zurich 1883 à l'ex-future expo tessinoise de 1998 en passant par Genève 1896, Berne 1914, Zurich 1939, Lausanne 1964 et l'échec de CH-9, Lausanne, Payot, 1991.

${ }^{11}$ O. L. A. Söderström, «Expo. 02: Exhibing Swiss identity», Ecumene, vol. 8, n 4, 2001, pp. 497-501.

${ }_{12}$ B. Crettaz, J. Michaelis-Germanier, Une Suisse miniature ou les grandeurs de la petitesse, Genève, Musée d'ethnographie de Genève, 1984, 185 p. 
Dans un ouvrage collectif consacré à l'Exposition de 1896 à Genève, l'historien Hans Ulrich Jost met en garde contre une lecture essentiellement «mythologique », psychologique et symbolique d'un tel objet historique, à ses yeux essentiellement économique, car «en privilégiant les aspects symboliques et esthétiques, elles [les méthodes anthropologiques] le transforment en un lieu devenu mythique où s'incarnent moins les valeurs matérielles qui le fondent et le structurent, que les forces spirituelles de la société, voire même l'âme du peuple. Le risque est ainsi pris de passer à côté de ce qui forme la colonne vertébrale d'un événement qui demeure, avant tout, une énorme foire moderne de la marchandise $»^{13}$.

En réalité, Jost réaffirme la primauté des «forces économiques », représentées par les milieux industriels qui s'incarnent dans une classe bourgeoise et dans les partis (alors) majoritaires. Fêtes, stands, pavillons, exhibition d'une Suisse rustique et laborieuse aux valeurs frugales ne donnent à voir qu'une «autoreprésentation » mystificatrice, masquant la réalité des inégalités et des disparités ainsi que celle des classes antagonistes, et nourrissant l'illusion d'une nation unie. La nation représentée, selon cette perspective, serait le produit d'un Etat lui-même au service de l'économie et des classes dirigeantes, une nation illusoire, affichée lors des Expositions nationales.

«Ici, il n'y a plus d'ouvriers ou de patrons, il n'y a que des Suisses!», dit à Meyer, l'ouvrier militant, son directeur de fabrique qui l'a invité à visiter l'exposition de Genève en 1896. L'ouvrier est réticent; il parle d'une «fête du capital qui ose ouvrir ses portes le jour de la fête des travailleurs!». Ce petit dialogue se trouve dans la bande dessinée éditée à l'occasion d'Expo.02, dans son récapitulatif de l'ensemble des Expositions nationales.

Aujourd'hui, la mondialisation et l'élargissement des marchés ont mené à une rupture du lien économie privée-expositions nationales, selon l'historien bâlois Georg Kreis; ces dernières sont désormais obsolètes: "l'économie n'a plus besoin d'expositions nationales comme devantures pour ses produits» et «la dimension nationale a largement disparu des préoccupations de l'économie ${ }^{14}$. La tenue d'Expo.02 d'une part, l'investissement affectif d'une grande partie de la populations dans ces manifestations de l'autre, montrent les limites d'une approche purement économiste. N'oublions pas que les expositions de 1939 et de 1964 ont accueilli chacune, en nombre de visiteurs, l'équivalent du double de la population de la Suisse ! Signe de l'adhésion dont elles peuvent être l'objet.

\section{UNE APPROCHE ETHNOLOGIQUE}

Les Expositions nationales ne se réduisent pas à une simple émanation des forces de l'économie; elles parlent un langage complexe, parfois contradictoire, qui va au-delà d'une simple information ou communication factuelle. Elles dévoi-

${ }^{13}$ H. U. Jost, «Les expositions nationales et leurs enjeux», in L. el-Wakil et P. Vaisse (dir.), Genève 1896. Regards sur une exposition nationale, Genève, Georg, 2000, p. 25.

${ }^{14}$ G. Kreis, «Expositions nationales 1800-2000. Quelles réponses à quels besoins?», in «Quand la Suisse s'expose. Les expositions nationales, $\mathrm{XIX}^{\mathrm{e}}-\mathrm{XX}^{\mathrm{e}}$ siècles », Revue Historique Neuchâteloise, janvier-juin 2002, Neuchâtel, p. 14-15. 
lent autant qu'elles dissimulent les crises et les aspirations du moment. Par leur expressivité même et leur caractère périodique (tous les vingt-cinq à trente ans en moyenne), elles sont les tentatives de figurer l'abstraction nationale et «l'être collectif» des citoyens, tel qu'il est imaginé par les élites et vécu par les visiteurs. D'où l'intérêt de l'étude des lieux, objets, figures et discours de telles manifestations observables dans un espace-temps délimité (le site spécifique, la durée éphémère) où choses et événements sont porteurs de sens et d'émotion.

Le fondement de l'approche ethnologique réside dans la relation singulière de l'ethnologue avec l'objet de son étude, relation qui est au cœur de son entreprise. La mienne n'échappe pas à cette particularité. Ma participation aux trois premières expositions n'est, bien entendu, que documentaire; celle aux trois dernières est, quant à mon rapport direct avec elles, imparfaite et lacunaire.

J'avais six ans en 1939. Mon père et ma mère visitèrent la «Landi »-Landesaustellung, appellation populaire de l'Exposition nationale de Zurich -, le premier en uniforme, mobilisé comme beaucoup de Suisses en ce début de la Seconde Guerre mondiale. L'exposition, qui s'ouvrit le 6 mai, ferma ses portes le 29 octobre. Mes parents me racontèrent leur visite avec une émotion visible, due aux circonstances et à l'atmosphère de ferveur entretenue autour des slogans d'unité nationale et de «défense spirituelle», conçus par les élites politiques et mis en scène plus spécialement sur le «Chemin des Hauteurs», une allée consacrée au «destin» de la Suisse, ornée de tous les drapeaux des communes suisses et dédiée « $\mathrm{Au}$ Peuple et à la Patrie». La devise de l'exposition était: «Un petit peuple créateur de grandes œuvres », exaltant l'effort et les accomplissements des Suisses. Cette émotion est à peu près le seul souvenir qui me reste de leur récit.

En revanche, ma participation fut plus active dans la préparation de celle de Lausanne en 1964. Le secteur «La Voie Suisse» qui devait «présenter le pays dans sa réalité » avait une subdivision intitulée «Un jour en Suisse », dans laquelle le géant Gulliver regardait la vie quotidienne des Suisses avec une distance qu'on pourrait dire ethnologique, une double distance même puisqu'à la différence de taille s'ajoutait celle du temps rendue visible par le costume XVIII ${ }^{\mathrm{e}}$ siècle porté par la gigantesque figure du héros de Swift!

Gulliver proposait aux visiteurs de répondre à un questionnaire touchant aux points sensibles d'une Helvétie non exempte de frilosité: condition de la femme, armée, inégalités sociales. 580000 personnes y répondirent ${ }^{15}$. L'exposition s'ouvrit quelques jours après mon départ pour l'Afghanistan. Je n'en ai donc visité que le chantier.

Sur les quatre sites d'Expo.02 enfin, j'ai été un flâneur assidu et quasi quotidien, mais un spectateur seulement, malgré une tentative avortée d'agrégation au groupe de réflexion d'un pavillon «Frontières» auquel aurait pu m'accréditer mon article «A propos des frontières intérieures de la Suisse ${ }^{16}$.

${ }^{15}$ Le questionnaire avait été préparé sur la base d'une étude préalable effectuée en 1962 par une équipe de sociologues et d'anthropologues dirigée par Isac Chiva, Ariane Deluz et Nathalie Stern. Les résultats ont été publiés et commentés par L. Boltanskki, Le bonheur suisse, Paris, Les Editions de Minuit, 1966.

${ }^{16}$ P. Centlivres, «A propos des frontières intérieures de la Suisse », in D. Fabre (dir.), L'Europe entre cultures et nations, Paris, Editions de la Maison des sciences de l'homme, 1996, pp.175-189. 
La ligne directrice de ces «terrains» est la question de savoir comment les diverses Expositions nationales ont cherché à répondre à la même interrogation sur l'objet «national » d'une façon différente, voire contradictoire, au gré des circonstances. Dans quelle mesure, quand et pourquoi une telle manifestation cesset-elle d'être concevable? Comment les deux dernières ont-elles intégré dans leur spectaculaires (re)présentations la critique et l'ironie, établissant ainsi une distance entre les visiteurs et ce qu'elles représentaient ${ }^{17}$.

Les premières expositions sont marquées par un contraste apparent entre la présentation de l'industrie et de ses produits, d'une part, l'hymne au travail, au patriotisme et à l'unité des Confédérés, de l'autre; la transformation des matières premières voisinant avec l'éducation du peuple, la scène patriotique faisant bon ménage avec la foire d'échantillon, dans une visée encyclopédique et didactique ${ }^{18}$.

En 1883, l'Exposition de Zurich fut inaugurée au son de «L'ouverture d'une nuit d'été» de Mendelssohn, et d'une Cantate à la gloire du Travail écrite par l'écrivain zurichois Gottfried Keller ${ }^{19}$. Elle fut précédée, et sa mise sur pied stimulée, par la Fête nationale de chant qui eut lieu à Zurich en 1880. Mais c'est l'inauguration de la ligne et du tunnel du Gothard un an auparavant qui en fut l'argument principal. Progrès, victoire sur l'obstacle des Alpes, réunion du Tessin aux autres cantons de la Confédération furent ainsi associés. L'ouvrage exprimait des symboles forts et, par le biais de la prouesse technique, unissait le principe de modernité à l'idée de patrie.

Chaque exposition fut ainsi marquée, jusqu'en 1964, par une prouesse ou invention nouvelle: électricité triomphante, force magique, en 1896 à Genève («la fée électricité»), percement du tunnel du Lötschberg (1912), débuts de l'aviation en 1914 (Exposition nationale à Berne), bathyscaphe à Lausanne en 1964. De 1896 à 1939, à Zurich de nouveau, la représentation de l'électricité passe de l'allégorie au symbole, de la nouveauté à l'omniprésence dans la vie quotidienne, une forme d'énergie spécifiquement suisse, voire composante de l'identité nationale, palliant l'absence de matières premières, de charbon en particulier - d'où l'électrification prioritaire des chemins de fer fédéraux -, une force issue du cœur même de la patrie, de l'eau des glaciers recouvrant ses montagnes ${ }^{20}$.

${ }^{17}$ Voir M. Heller: «L'Exposition et la vie», in ImagiNation. Le Livre officiel d'Expo.02, Lausanne, Payot, 2002, p. 291.

${ }^{18}$ Les Suisses dans le miroir, op. cit., p. 19.

19 Oui, pays de nos pères, c'est là ta victoire

Tu es l'égal des autres nations!

Laisse l'ivresse s'en aller.

Ta richesse te viendra de son seul travail»

(Chant solennel d'ouverture, Zurich 1883)

${ }^{20}$ P. Kaenel, «Vendre l'invisible: électricité et publicité en Suisse», Zeitschrift für Archäologie und Kunst 58, Hft 3/1, 2001, pp. 229-240. 


\section{LA SUISSE AUX EXPOSITIONS UNIVERSELLES DE PARIS, SON VILLAGE ET SON PANORAMA}

La double mission donnée par leurs initiateurs aux Expositions nationales du $\mathrm{XIX}^{\mathrm{e}}$ siècle: instruire les visiteurs suisses en leur présentant leur propre pays dans son unité complexe d'une part, montrer aux visiteurs étrangers la capacité de production industrielle de la Suisse et les réalisations de son savoir-faire, aussi bien artistique qu'industriel, de l'autre, se retrouve à peu de chose près, pour un public différent, dans les pavillons suisses des Expositions universelles à Paris.

L'excellence industrielle, voilà ce que la Suisse veut montrer aux visiteurs des grandes Expositions de 1867 et 1889 . Un pays qui, par la production de ses fabriques, peut se montrer à l'égal des grandes nations modernes. «Les villes suisses », écrit en 1867 Victor Cosse, « sont des centres d'industrie, de commerce et de progrès... ${ }^{21}$, un pays qui rivalise, poursuit-il, avec les nations puissantes. Sa recette, c'est le travail, l'indépendance, la liberté. Pas d'industrie de luxe ou d'objets d'art. Ce qui domine, c'est l'esprit pratique et l'activité laborieuse, et Victor Cosse anticipe ainsi l'hymne au travail de l'Exposition nationale de 1883 à Zurich. Il cite ensuite la galerie des machines avec ses moteurs, ses machines à vapeur, ses engins de navigation, l'horlogerie et l'industrie textile. La réussite de l'industrialisation s'affirme encore davantage à l'Exposition universelle de 1889; «les cantons se sont couverts de fabriques», «on s'est américanisé autant qu'il a été possible», s'exclame Léon Pradel ${ }^{22}$. «Les Suisses », surenchérit un autre chroniqueur, Léon Dussert, «ont maintenant des machines-outils pour toutes choses, comme les Américains, et fabriquées par eux-mêmes $»^{23}$.

Restent en arrière les Beaux-arts, hélas qui, au même titre que la Science et l'Industrie, attestent le progrès et la réussite des Etats-Nations sur la voie de la civilisation. Comment expliquer, se demandent les chroniqueurs des Expositions, le décalage entre l'idéal de liberté et de démocratie, la réussite industrielle, d'une part, et la médiocrité des productions artistiques, de l'autre? L'Art est-il incompatible avec la frugalité laborieuse des libres enfants de l'Helvétie?

En 1867 déjà, Victor Cosse concluait sa chronique intitulée Les Beaux-Arts de la Suisse, par ces mots «Et ne serait-on pas amené à conclure que si la démocratie féconde et développe la force, l'énergie, les sentiments généreux, elle est sans influence sur l'art et sur l'imagination?» ${ }^{24}$.

Mêmes réserves en 1889: «On s'est souvent demandé pourquoi nulle école d'art ne s'est anciennement fondée en cette terre libre, et pourquoi nul grand artiste ne s'y est développé. Faut-il attribuer, comme on l'a fait, cette pénurie pittoresque, cette disette de maîtres à l'écrasante beauté des Alpes qui rend impossible toute imitation et décourage l'artiste par la comparaison», se demande

${ }^{21}$ V. Cosse, «Les Beaux-arts de Suisse», in L'Exposition universelle de 1867 illustrée, I, Paris, 1867 , pp. 227-231. p. 391.

${ }^{22}$ L. Pradel, «L'horlogerie suisse, III », Revue de l' Exposition universelle de 1889, I., Paris, 1889,

${ }^{23}$ L. Dussert, «Promenade aux sections étrangères », in Revue de l'Exposition universelle de 1889, II, 1889, p. 236.

${ }^{24}$ V. Cosse, «Les Beaux-arts de Suisse», op. cit., p. 231. 
Léon Pradel ${ }^{25}$. «Les arts, dit-il, ne fleurissent qu'à l'abri d'une paix assurée, du luxe et du goût entretenus par la richesse ${ }^{26}$; et de se moquer «de ces broderies représentant Guillaume Tell ajustant sa pomme, des montagnes coiffées de sapins, de bergères à grands chapeaux. [...]. En a-t-on vu de ces glaciers peints sur bois blanc et de ces bois sculptés dignes du bazar à treize sous, et de ces boîtes à musique! ${ }^{27}$. «La Suisse était ballottée entre l'imitation et une naïveté confinant à la niaiserie». Mais le présent est encourageant, le goût s'améliore «dans l'avenir, elle réussira mieux encore », écrit Pradel ${ }^{28}$. Même optimisme mesuré chez Léon Dussert: «Il ne reste plus aux Suisses qu'à s'affiner un peu le goût, mais voici qu'ils ont déjà des artistes, et nul doute qu'ils ne finissent par avoir un art ${ }^{29}$. L'accomplissement de l'Etat-Nation repose aussi sur un Art national reconnu et reconnaissable.

Pour les commentateurs français, la Suisse, appelée à se présenter elle-même dans le cadre des expositions universelles, souffre donc d'une sorte de déficit d'art, qui est en fait un déficit identitaire. L'égale des grandes nations par le développement industriel, la Suisse n'a pas d'art à la fois national et accompli, à l'instar de la France, de l'Italie ou de la Suède.

L'identité nationale, au profit de laquelle les expositions, ou plutôt les élites qui les mettent sur pied, enrôlent culture et Beaux-arts, comme le montre Pascal Ruedin dans sa thèse, est dans le cas de la Suisse, dans le domaine de l'Art, pas encore parvenue au niveau de ses autres réalisations ${ }^{30}$. Chez les commentateurs des expositions de 1867, 1889 et 1900, domine une vision évolutive; la Suisse, encore déficiente, est en progrès dans les expositions de 1896 et de 1900.

En 1900 cependant, l'accent porte moins sur la Suisse des machines et du progrès industriel que sur le salon de l'art suisse et, avant tout, sur le Village suisse importé de l'Exposition de Genève de 1896, avec le gigantesque panorama des Alpes bernoises. Village et panorama livrent un message identitaire enfin explicite. Les chroniqueurs cherchent désormais dans l' «Art suisse»l'écho de son essence alpestre, que le Village et le panorama illustrent par excellence.

En 1900, le public français et international aspire moins à admirer une nation créatrice de machines-outils, la réussite d'un petit pays apportant sa contribution à la civilisation et au progrès, qu'à contempler un mythe, celui de l'harmonie du village alpestre dans son majestueux décor. Désormais à Paris comme à Genève, où Village suisse et Panorama avaient obtenu un énorme succès, la vogue des villages typiques d'un passé idéalisé ne se démentit pas du dernier tiers du XIX siècle à la veille de la Seconde Guerre mondiale. L'image d'une Suisse alpestre, miniaturisée à la taille d'une petite communauté villageoise (avec quelques maisons patriciennes des villes de l'ancienne Confédération) aux mœurs simples, éprise de liberté et de costumes folkloriques, représente une des figures régressives et nostalgiques de la Suisse pour les citadins des pays industriels ${ }^{31}$.

${ }^{25}$ L. Pradel, «L'horlogerie suisse, III », op. cit.

${ }^{26}$ Ibid., p. 391.

27 Ibid.

28 Ibid., pp. 391-392.

${ }^{29}$ L. Dussert, «Promenade aux sections étrangères », op. cit.

${ }^{30}$ P. Ruedin, La participation des artistes suisses aux expositions universelles de Paris (18551900). Problèmes d'une représentation nationale, Thèse soutenue en 2004, (à paraître). 
A Paris, Gustave Regelsperger, qui couvre la Suisse à l'Exposition de 1900, insiste sur la vérité, l'exactitude du village: «Une image réduite, mais fidèle de la Suisse toute entière, écrit-il, pas une Suisse de fantaisie [...] la vraie Suisse, avec ses montagnes, ses cascades, ses pâturages, ses vieux édifices [...], toute une population revêtue des costumes si gracieux et si variés des divers cantons », tout cela reproduit avec «exactitude et sincérité » ${ }^{32}$.

Par rapport à l'Exposition, le Village suisse est situé un peu en dehors, en bordure du Champ de Mars, au carrefour de l'avenue de Suffren et de celle de la Motte-Piquet. Il ne s'agit pas d'une mise à l'écart, mais plutôt d'un effet de réel par lequel le Village, hors du périmètre éphémère de l'Exposition, occupe un espace plus étendu que celui dont il aurait pu bénéficier à l'intérieur, d'un espace en quelque sorte «permanent», garantie de durée qui se perpétue aujourd'hui sous la forme d'un quartier d'antiquaires.

L'immense «Panorama des Alpes Suisses », placé dans les entrailles du décor artificiel du Village suisse, est dû aux peintres vaudois et genevois Eugène Burnand, Auguste Baud-Bovy et Francis Furet. C'est «le plus grand paysage jamais peint...» dit Philippe Kaenel, qui lui a consacré un chapitre dans son livre sur Eugène Burnand: dix-sept mètres de haut sur cent quinze de long. C'est un sommet du «genre alpestre, considéré comme 'national' et helvétique par excellence ${ }^{33}$. L'œuvre, que son commanditaire Charles Henneberg exhibe dans de nombreuses occasions: Vincennes (1892), Chicago (1893), Anvers (1894), Genève (1896) et Paris, est détruite par un ouragan à Dublin en 1903.

Le Panorama est un complément du village souvent présenté comme une miniature, un modèle réduit d'une Suisse alpestre et archaïque. Un complément qui joue, lui, sur l'immensité et l'aspect spectaculaire du trompe-l'œil. Ce «vrai» Disneyland Helvétique offre un «horizon national» suisse hors limites. Débordant son motif, les Alpes bernoises, il semble embrasser l'ensemble de la Suisse des Alpes au Jura, et ces montagnes deviennent sur la toile un monument identitaire à la fois symbole des libertés helvétiques et théâtre de l'histoire nationale, poursuit Kaenel ${ }^{34}$. Le paysage, presque sans figures humaines, prend la place de la grande peinture d'histoire, comme symbole de la patrie. La petitesse de la Suisse, le modèle réduit du village y est transfiguré par la majesté grandiose du paysage.

Grâce à des artifices tels des écrans peints disposés entre la peinture et le spectateur, ce dernier a l'impression d' «y être» véritablement - du moins c'est ce que disent les témoins - non pas comme un visiteur arpentant une Suisse en réduction, mais comme un voyageur presque écrasé par le cirque des sommets helvétiques. L'illusion triomphe. Le Panorama semble s'étaler par-delà l'opposition nature/peinture, la différence entre un décor bidimensionnel et la nature tridimensionnelle.

${ }^{31}$ B. Crettaz, J. Michaelis-Germanier, Une Suisse miniature ou les grandeurs de la petitesse, op. cit.

32 G. Regelsperger, «Le village suisse à l'Exposition de 1900 », in L'Exposition de Paris (1900), première partie, Paris, Montgredien et Cie, 1900, p. 281.

33 P. Kaenel, Eugène Burnand (1850-1921), peintre naturaliste, Lausanne, Musée cantonal des Beaux-Arts, 2004, p. 67.

${ }^{34}$ Ibid., p. 83. 
Par le Panorama, le pays des libertés démocratiques et les Beaux-arts pourraient à première vue se réconcilier enfin, et répondre ainsi aux attentes dédaigneuses des critiques des Expositions universelles antérieures, mais au prix d'un doute quant aux frontières entre l'art et la réalité, donc aux dépens de son statut d'œuvre d'art. La peinture nationale et identitaire court le risque d'être ravalée à un «genre méprisé » par les peintres, un genre somme toute pas si éloigné d'une attraction foraine.

\section{LA NATION SUISSE}

La notion de «nation suisse», un peu inhabituelle aux oreilles helvétiques, va sans dire sous la plume des chroniqueurs français des Expositions universelles de 1867 et 1886 , en relation avec ce qui est désigné comme «caractère suisse » et «art suisse». Nulle part sous leur plume il n'est fait mention du multiculturalisme suisse et du pluralisme linguistique et religieux. La diversité mentionnée s'inscrit dans le pittoresque des paysages et des costumes; le cadre descriptif et explicatif et les stéréotypes se réfèrent à un Etat-Nation implicitement homogène au même titre que les Pays-Bas, la France ou la Suède.

En réalité, la nation n'est guère explicite dans les expositions nationales en Suisse, pas plus que dans la vie politique en général. Les expositions, elles, sont liées, au XIX $\mathrm{X}^{\mathrm{e}}$ siècle, à bien des facteurs politico-économiques, dont le développement des communications intercantonales et l'établissement d'un programme de compromis politique entre l'ancienne indépendance des cantons et le lien fédéral renforcé établi par les Constitutions de 1848 et de 1874 . Le «national» est conforme aux aspirations du parti dominant qui se veut celui du progrès et de tous ceux qui, à l'occasion des fêtes patriotiques et autres rencontres fédérales (tir, sport, chant...), considèrent qu'ils ont en commun une «patrie suisse». Le mot «nation» justement ne figure que dans le préambule des Constitutions fédérales susmentionnées. Il a curieusement disparu de celle, toute nouvelle, de 1998 ! Cette dernière, conformément à l'usage du discours politique, utilise les termes de peuple suisse ou de Confédération. La notion figure sous sa forme adjectivale dans «Conseil national», qui réunit les représentants élus du peuple, ou dans «banque nationale»; mais la réunion des deux chambres se dit «parlement fédéral ${ }^{35}$. A la différence des ouvrages français décrivant les sections suisses des expositions universelles, je n'ai guère trouvé d'occurrence du terme dans les documents et discours publiés à l'occasion des expositions nationales suisses de 1883 à 1964. Pays, patrie, peuple suisses, voilà les termes utilisés.

Depuis plus d'un demi-siècle, la Suisse est le seul pays d'Europe à organiser des expositions au nom de la nation, preuve d'un enjeu qui va au-delà de la présentation des capacités de la Suisse dans les domaines de l'industrie, de l'agriculture et des arts, et au-delà de la volonté d'instruire et d'informer les citoyens. Elles sont aussi des manifestations dont la signification, politique, va au-delà d'une foire d'échantillon. Qu'y a-t-il donc de «national» dans les expositions

${ }^{35}$ P. Centlivres, D. Schnapper, «Nation et droit de la nationalité suisse». Pouvoirs, 56, 1991, p. 155. 
nationales, si ce n'est la volonté de montrer à un public de citoyens auxquels «manquent les unités essentielles de langue et de race», comme l'écrit Gonzague de Reynold ${ }^{36}$ dans l'ouvrage collectif consacré à l'Exposition de 1939, ainsi qu'aux visiteurs étrangers, la réalité, ou plutôt le processus de formation d'une entité nationale? Ses dimensions et ses composantes, font l'objet d'une interrogation poursuivie et reformulée de 1883 à 1964. Cela ne va pas sans dire, malgré l'image unifiée autant que performante que la Suisse a pu donner d'elle-même dans les expositions universelles à l'Etranger.

L'inexistence de la «nation ethnique» place la nation de volonté devant un déficit d'essence, celle de l'origine partagée, de la fiction d'une parenté charnelle, d'une culture homogène. D'où l'obligation d'établir à chaque fois son existence: exercice démonstratif, et d'en appeler à l'unité malgré divisions, hétérogénéité et inégalité : exercice performatif.

La mention de l'unité et de la cohésion nationale est donc récurrente dans les Expositions nationales, depuis l'évocation de «l'esprit d'harmonie» par le conseiller fédéral Numa Droz ${ }^{37}$ et l'appel au "peuple tout entier», «l'égal des autres nations » de Gottfried Keller lors de l'inauguration de celle de Zurich en 1883, jusqu'au mandat du Conseil fédéral pour Expo.02 $2^{38}$.

Dans un court article intitulé Le portrait introuvable, j'avais abordé un certain nombre de motifs récurrents des Expositions nationales, fonctionnant comme des métaphores de la Suisse: village, île flottante, petitesse ${ }^{39} \ldots$

J'aimerais relever ci-après un certain nombre de thèmes «identitaires » ou symboliques, réinterprétés de manifestation en manifestation.

\section{FÊTE, SPECTACLE. FÊTE OU SPECTACLE?}

Dans un article consacré à ce qu'elle appelle les Swiss political rituals, soit les célébrations telles que les fêtes de tir ou les fêtes nationales, Regina Bendix insiste sur l'écho relativement faible que recueillent, à la différence des premières, les cérémonies organisées par l'Etat fédéral. «The Swiss have little emotional investment in the nation ${ }^{40}$. Il est vrai que la fête du premier août, qui est censée commémorer la fondation de la Confédération, ne suscite pas en temps normal la ferveur populaire d'un 14 juillet chez nos voisins français, avec défilé militaire et bal populaire. Ce sont les fêtes locales ou régionales, avec un fort accent corporatiste,

${ }^{36}$ G. De Reynold, «La formation et l'évolution du peuple suisse», in La Suisse vue à travers l' Exposition nationale. Ouvrage collectif présenté dans les quatre langues nationales, 4 vol., Zurich, Editions Atlantis, vol. 1, 1940, p. 112.

37 Voir G. Kreis, «L'Exposition nationale de 1883 » in Les Suisses dans le miroir. Les expositions nationales suisses. De Zurich 1883 à l' ex-future expo tessinoise de 1998, en passant par Genève 1896, Berne 1914, Zurich 1939, Lausanne 1964 et l'échec de CH-91, Lausanne, Payot, 1991, pp. 132-137.

${ }^{38}$ Forum helveticum, «Expo. 02 et cohésion nationale», Forum helveticum, Lenzburg, 2003, p. 6.

39 P. Centlivres, «Le portrait introuvable: la Suisse des expositions nationales », op. cit.

${ }^{40}$ R. Bendix, «National sentiment in the enactment and discourse of Swiss political ritual», American Ethnologist 19/4, 1992, p. 784. 
qui recueillent la ferveur populaire, tels les carnavals de Bâle ou de Brigue, les montées à l'alpage et les cortèges historiques qui se multiplièrent au XIX ${ }^{\mathrm{e}}$ siècle, dans un mouvement général d'invention de la tradition.

Dès celle de Zurich en 1883, et surtout à Genève en 1896, les organisateurs des Expositions nationales ont compris qu'il était important de donner à ces manifestations un éclat populaire allant au-delà de la distribution de médailles par un jury officiel. La liesse populaire rassemble, et les spectacles de type «Festspiel» qui mettent en scène l'histoire héroïco-mythique de la Confédération, les fêtes folkloriques et les concerts populaires doivent divertir et pas seulement instruire la foule des citoyens rassemblés.

On sait que les grandes fêtes de gymnastes, de tireurs ou de chanteurs ne sont pas étrangères à l'élan qui est à l'origine des Expositions nationales: le tir évoque le pays où «chaque enfant naît soldat» et où les citoyens gardent leur arme d'ordonnance à leur domicile. Le chant passait au $\mathrm{XIX}^{\mathrm{e}}$ siècle pour une spécialité helvétique; en 1901, on estimait le nombre des membres actifs des sociétés de chant à 300000 environ!

La mise en scène des Expositions et les rituels patriotiques auxquels elles ont donné lieu furent donc l'occasion de manifestations qui oscillent entre la fête à participation populaire et les spectacles artistiques exécutés par des professionnels. Dans la première, les participants recréent une «communitas », l'espace de quelques heures arrachées au quotidien. Les spectacles, eux, sont liés à la solennité d'une inauguration ou aux préoccupations de l'élite, et sont marquées du sceau de l'officialité, à moins qu'ils ne soient censés élever et instruire le peuple, à partir du répertoire appartenant à la culture «cultivée».

Les organisateurs des premières expositions nationales penchaient du côté «solennité » et «folklore patriotique»: cantates officielles, hymnes au travail ou spectacles sur fond de nostalgie de la vie alpestre. Fêtes débridées, populacières, non! On craignait les débordements. Selon Les Suisses dans le miroir, «A Zurich, le côté festif était réduit: un concert par jour et une loterie. Les visiteurs étaient des fidèles venus là pour apprendre et participer au culte de la machine et du progrès ${ }^{41}$. A Genève en 1896 en revanche, malgré la voix puritaine des sociétés de tempérance qui voulaient bannir débits de boissons et divertissements profanes, l'exposition donna une importance considérable aux activités festives, en multipliant les concours de poésie, les concerts classiques, ainsi que les manifestations folkloriques. Cantates solennelles, poèmes et concerts faisaient partie du projet genevois de proposer aux visiteurs les manifestations d'une «culture suisse» et d'un «art suisse», avec le folklore alpestre du Village et les cantates patriotiques, d'un côté, le Palais des Beaux-Arts abritant les œuvres de peintres et de sculpteurs helvétiques, de l'autre. Les constructions de circonstance elles-mêmes devaient contribuer à cette démonstration d'esthétique helvétique par l'élaboration d'un «style suisse», à base de galeries de bois et de clochetons.

Chaque semaine, le Village fut ainsi le théâtre de réjouissances populaires, allant des concours de tir à l'arbalète aux représentations de danses villageoises et aux concerts religieux dans l'Eglise. Elles devaient contribuer à démontrer un patrimoine commun.

${ }^{41}$ Les Suisses dans le miroir, op. cit., p. 33. 
Le genre du Festspiel, festival populaire, ou plutôt, comme le dit le livre-souvenir, «sorte de cortège où les chœurs miment l'action», et dont le type le plus achevé est, selon lui, la Fête des Vignerons qui se tient tous les vingt-cinq ans à Vevey, est un poème lyrique en action. Au Village suisse de Genève, justement, une société chorale alémanique chante un poème intitulé «Scènes des moissons », en deux parties: «Le Départ pour l'Alpe, troupeau en tête» et «La Fête sur la montagne ». Le Festspiel est le modèle de ces divertissements qui allient l'unanimisme de la fête (les acteurs sont des amateurs) et le spectacle réactivant un passé imaginé ainsi que le cycle éternel des activités des champs et de l'alpage.

Que ce soit à la «Landi» de Zurich en 1939 ou à la Fête des Vignerons de Vevey lors de son édition de 1999, la modernisation du contenu exigée par les organisateurs qui trouvaient le spectacle et ses harmonies vieux jeu, fut rejetée par le public. La volonté du metteur en scène François Rochaix de monter un Festspiel mondialisé, sans références aux thèmes rustiques et historicisant habituels, contribua à l'échec du spectacle d'ouverture d'Expo.02.

A Genève en 1896, les festivités populaires avaient été encadrées par les cérémonies officielles de l'ouverture et de la clôture, et la cantate d'inauguration, dont les paroles étaient dues à Jules Cougnard, reprenaient les exhortations au peuple suisse et le thème du travail de l'hymne inaugural de Zurich en 1883.

A Berne en 1914, l'inauguration fut marquée, là aussi, par un contraste entre la solennité officielle avec son défilé de personnalités politiques en habits noirs et chapeaux haut-de-forme, jugé ennuyeux par la presse locale, et le cortège folklorique des représentants des associations locales en costumes «historiques », suivis des membres des sociétés de tir, de chant et de gymnastique, une association typique des festivités patriotiques ${ }^{42}$. En revanche, le programme d'animations fut l'objet d'un vif débat autre les partisans d'un «parc de plaisir» avec divertissement tous publics: montagnes russes et pistes de danses, et ceux en faveur de récréations plus austères, influencées par les pressions croissantes du Heimatschutz (préservation du patrimoine), fondé en 1906, décidé à lutter contre la «barbarie culturelle» et le matérialisme; milieux économiques contre gardiens de la moralité helvétique, besoin de rentabilité contre devoir d'édification.

A Berne enfin, l'ambition d'une «culture suisse», comme élément de l'identité nationale tendit à se replier sur les traditions populaires, alors que la tentative de définir et d'exposer un art national s'avéra définitivement irréalisable ${ }^{43}$.

Je mentionne rapidement le spectacle officiel de la Landi de 1939 «Le Triomphe de l'esprit helvétique», bien analysé par Pierre-Alain Tschudi ${ }^{44}$. L'Exposition se déroula dans un contexte de guerre imminente, attisant la ferveur patriotique. La prise de distance par rapport aux régimes autoritaires voisins se mua en repli sur les «valeurs helvétiques», présentées comme la «défense

${ }^{42}$ C. Jörg, «Die Schweizerische Landesaustellung 1914 in Bern: zwischen Fortschrittsglaube und Kulturkritik», in Expos.ch. Idées, intérêts, irritations, Berne, Archives fédérales suisses, 2000, pp. 131-149.

43 Ibid., p. 144.

${ }^{44}$ P.-A. Tschudi, «Die Konstruktion der christlichen Nation im offiziellen Festspiel der Landi $39 »$, in Expos.ch. : idées, intérêts, irritations, op. cit., pp. 179- 199. 
spirituelle du pays», impliquant la nécessité de défendre «l'âme suisse » contre «les influences étrangères ${ }^{45}$. Le spectacle connut un succès sans précédent et fut applaudi par 140000 spectateurs au cours de différentes représentations. Il s'agissait d'une dramaturgie prophétique, allégorique et morale où était exaltée la spécificité suisse autour des valeurs de liberté, de neutralité et d'humanitarisme, nourrie des principes chrétiens. La diversité linguistique et culturelle, ainsi que la nécessité de surmonter les divisions entre Confédérés, y étaient affirmées. Le spectacle a été analysé comme un compromis entre les grandes liturgies totalitaires et la mise en scène des libertés démocratiques symbolisées par les Landsgemeinde. La recherche d'une «suissité » recélait un danger d'ordre moral autoritaire, mais était contrebalancée par l'éloge du pluralisme.

Toutes autres furent les festivités à Lausanne en 1964. Le précédent de la Landi apparaissait à beaucoup comme insurpassable. Le contexte était en outre bien différent en ces années de «surchauffe» économique; le « repli » apparaissait appartenir à une époque révolue à laquelle devait succéder l'ouverture sur l'Europe et le monde. L'Exposition voulut donc se démarquer des expositions antérieures; «ni foire, ni fête, ni rite, ni musée », écrit Frédéric Sardet, archiviste de la Ville de Lausanne. «L'expo 64 a été conçue comme une confrontation directe à la 'tradition'» ${ }^{46}$. Pourtant, l'opposition habituelle solennité/festivité populaire était bien là! Le représentant du Conseil fédéral s'opposa à la publication des réponses au questionnaire de Gulliver en arguant du manque de sérieux d'une consultation effectuée dans une atmosphère festive, donc relâchée, hors d'un contexte sérieux propice à la consultation des citoyens.

Comme précédemment, et conformément au sentiment d'appartenance infranationale, qui prime, émotionnellement, sur l'appartenance abstraite à l'Etat fédéral, les journées cantonales, les fêtes des jodleurs et des costumes suisses, remportèrent un grand succès populaire.

\section{ARCHITECTURE : UN «STYLE SUISSE»?}

Les partisans d'une mise en scène d'une «culture suisse» en accord avec une classe moyenne désireuse de montrer les progrès de la nation rêvaient d'expositions bâties selon un «style suisse». Parlant des architectes et des ingénieurs responsables des constructions de l'Exposition de Genève, le Journal de Genève s'écriait: "Ils ont trouvé un style vraiment suisse, adapté une construction en pierre et en bois, sans tomber dans le genre conventionnel du chalet; c'est le style des vieilles villes de la vieille Suisse, avec leurs toits aux courbures multiples, leurs avant-toits, leurs tourelles, leurs charpentes multicolores et leurs galeries couvertes $»^{47}$.

${ }^{45}$ U. Im Hof, Mythos Schweiz. Identität-Nation-Geschichte. 1291-1991, Zurich, Neue Zürcher Zeitung, 1991, pp. 947-948.

${ }^{46}$ F. Sardet, «Organiser l'Expo 64 : espace, argent et pouvoirs», in Expos.ch. : idées, intérêts, irritations, op. cit., p. 221.

${ }^{47}$ Cité par S. Lob-Philipp: «L'architecture des pavillons de l'Exposition nationale de 1896. Problèmes stylistiques », in Leïla el-Wakil et Pierre Vaisse (sous la dir.), Genève 1896. Regards sur une exposition nationale, Genève, Georg, 2000, p. 93. 
La commission des constructions responsable de l'invention d'une « architecture nationale» avait renoncé, pour le Palais des Beaux-Arts, le monument phare de l'Exposition, à une construction rustique en bois, jusqu'alors considérée comme typique de la Suisse pour s'inspirer des tours et maisons de pierre des anciennes villes suisses, en une synthèse historicisante. Mais cette commission, chargée d'exprimer par l'architecture de l'exposition quelque chose de l'identité nationale, s'est bien vite rendue compte que la solution choisie ne saurait être considérée comme «typiquement suisse», et que le terme «style suisse» ne correspondait à rien de clairement défini, d'où l'appellation plus vague de «genre suisse». Le Village suisse, ainsi que l'architecture «genre suisse» du Palais des Beaux-Arts, qui abritait lui-même, dans sa partie «moderne», les œuvres de quatre cents artistes suisses ou établis en Suisse, affirmait cependant aux yeux du monde, du moins des 2.300 .000 visiteurs, l'existence d'une «culture suisse», et dans le registre élevé de la grande peinture, et dans le registre traditionnel, historique et populaire.

Style ou genre architecturaux suisses furent du reste abandonnés dans les expositions ultérieures, et confinés au «Village», qui se perpétue, ou plutôt renaît en 1914 à Berne, mais un village désormais plus homogène, modernisé, vitrine de l'agriculture et de l'industrie laitière, tout en gardant son exemplarité paysanne liée aux valeurs autochtones.

L'Exposition de Berne vit apparaître une attitude de méfiance à l'égard des influences étrangères et du mauvais goût «importé» et laisse percevoir les signes d'un fossé séparant les Romands des Alémaniques.

La population étrangère en augmentation inquiète une partie de l'opinion; les étrangers formaient alors quinze pour-cent de la population environ, dont quarante pour-cent d'Allemands actifs dans l'économie et la culture. Beaucoup de Romands en trouvaient l'influence excessive, voire dangereuse, d'où le mauvais accueil qu'ils firent à l'architecture de l'Exposition de 1914, inspirée par le néoclassicisme allemand et par le style de l'Exposition universelle de Munich en 1910. Ils éprouvaient la nostalgie de l'historicisme et de l'helvétisme de celle de Genève.

La Landi en 1939 revit le Village, synthèse de plusieurs types régionaux. Son succès participait là aussi du repli sur le «visage aimé de la Patrie», et sur la réhabilitation d'une paysannerie désormais nettement minoritaire (vingt-cinq pourcent de la population), une réhabilitation commencée déjà vers 1900 . On put voir à la Landi un nouveau panorama (110 sur 6,5 m.) dû au peintre Hans Erni, alliant l'univers technique: conduites forcées et locomotive aérodynamique, aux scènes les plus traditionnelles: Landsgemeinde et procession religieuse, articulant deux types d'énergie collectives et deux formes de solidarités ${ }^{48}$. Mais cette fois il ne s'agissait plus de promouvoir le paysage des Alpes comme alliant beaux-arts et identité nationale, mais d'un tableau allégorique où l'on trouvait les grands thèmes d'un humanisme progressiste.

Panorama à nouveau à Expo.02, sur le site de Morat, à l'intérieur du monolithe de Jean Nouvel. La bataille de Morat (22 juin 1476), brossée en 1894 par le peintre allemand Louis Braun, restaurée, déroulait son ample scénario, tableau historico-

${ }^{48}$ P. Kaenel «Vendre l'invisible: électricité et publicité en Suisse», op. cit., pp. 233-234. 
patriotique fin de siècle rappelant les Expositions du XIX ${ }^{\mathrm{e}}$ siècle. Elle représentait le volet rétro d'une triple représentation panoramique, les deux autres consistant en la projection changeante et fragmentée de photographies puisées dans les fonds privés, donnant à voir «le vrai visage de la Suisse», et enfin dans le panorama «réel» du paysage de Morat visible à travers les ouvertures du monolithe. En proposant ce triple regard, Expo.02 à Morat assumait, tout en le relativisant, le passé panoramique des Expositions nationales.

\section{L'ÉPHÉMÈRE ET LE PERMANENT}

A Lausanne, lors des phases préparatoires de l'Exposition de 1964, un groupe de jeunes architectes proposa de faire appel à Le Corbusier pour un projet urbanistique révolutionnaire: l'Exposition serait l'occasion d'une expérience audacieuse, préfiguration de la Suisse de demain. L'espace prévu pour l'Exposition serait aménagé selon une articulation exemplaire des zones agricoles, urbaines et industrielles, créatrice d'un nouvel espace de vie sociale et culturelle. A la place du traditionnel «Dörfli » toujours renaissant: une ville nouvelle destinée à durer.

Ainsi apparaissait clairement - ce n'était pas la première fois - la contradiction propre aux expositions, entre le permanent et l'éphémère, l'utopie et les contraintes imposées, l'insularité des expositions et l'espace préexistant. Le projet en tant que tel fut écarté, même si l'exposition aboutit à des aménagements durables. Les organisateurs déclarèrent qu'une exposition à l'échelle $1 / 1$, destinée à une durée permanente, en ne distinguant pas entre l'événement temporaire et la ville, entre visiteurs et habitants, y perdrait son caractère de manifestation d'exception $^{49}$.

Les Expositions n'échappent pas à la tentation de la durée. Elles laissent parfois, de beaux restes, en Suisse comme à Paris: il subsiste quelques chalets du fameux Village à Genève et à Lausanne. Le caractère passager et la brièveté sont indissociables de la fête et de l'utopie sous forme d'esquisses, de prototypes destinés à disparaître «L'exposition est éphémère, la construction de son décorum et la prise de possession d'un lieu, même limitée, autorise toutes les audaces, toutes les innovations et toutes les extravagances $»^{50}$.

Décors, toiles peintes, maquettes, bâtiments provisoires sont la règle davantage que les constructions durables. Ces dernières existent cependant et subsistent comme des fragments isolés survivant au naufrage programmé de la manifestation.

A Expo.02 sur le site de Morat, on pouvait voir, sur la rive du lac, le fameux mésoscaphe de Jacques Piccard, ou plutôt son épave rouillée, rappel pitoyable de - ou hommage à - ce qui avait été l'attraction de l'Exposition de Lausanne en 1964. On pouvait contempler des constructions de l'Exposition actuelle, anticipant par la rouille qui les rongeait déjà - l'effet était voulu - leur prochaine

${ }^{49}$ F. Sardet, «Organiser l'Expo 64: espace, argent et pouvoirs», in Expos. ch. : idées, intérêts, irritations, op. cit., pp. 219-235.

${ }^{50}$ M. Sardi, C. Zimmermann, «L'Exposition nationale suisse: de la représentation à l'image», Genève, Département de sociologie, [typoscrit non publié], 1985, p. 152. 
décrépitude et l'inéluctable destin de l'Exposition toute entière, conformément au thème du site: Instant et Eternité.

Plus que d'autres, l'Expo.02 fut conçue comme éphémère, condamnée non seulement à disparaître, mais aussi à ne pas laisser de traces, à remettre les cinq sites «en l'état», c'est-à-dire comme s'il ne s'était rien passé. Les interventions des défenseurs de l'environnement ne suffisent pas à expliquer cet effacement. Certes, il a fallu rassurer une partie de l'opinion, réticente à l'idée des rives des trois lacs durablement défigurés, mais tout se passe comme si l'on avait affaire à deux conceptions aussi contrastées que complémentaires: celle d'un environnement achevé, intangible, qui ne saurait être modifié, auquel rien ne saurait être ajouté sous peine d'un irrémédiable gâchis, et celle de l'incertaine, changeante et évanescente «représentation du multiculturalisme et de l'identité suisse», pour reprendre les termes de Nelly Wenger, présidente de la direction générale d'Expo.02 (Allocation à l'Université de Neuchâtel, 4 novembre 2000), une représentation appartenant éminemment à l'instantané et non à la durée.

Sa survie appartient par conséquent et aux archives et à la mémoire. Pour les premières, sept cents mètres linéaires sont désormais confiés aux souterrains des Archives fédérales. Pour la seconde, le temps des commémorations et des associations de visiteurs nostalgiques est venu, ainsi que les livres-souvenirs. De l'architecture audacieuse de l'Expo.02 «Tout a disparu, il reste un livre», dit un prospectus d'éditeur. Cet ouvrage est un compte-rendu des audaces techniques et monumentales des quarante pavillons d'exposition, décrites davantage comme «un rêve un peu fou », un monument poétique, utopique, que comme des structures de tôles brutes, de toiles patinées, de béton et de bois. Son évanescence est symbolisée par le Nuage d'Yverdon-les-Bains, qui vaporisa l'eau du lac de Neuchâtel à partir de 30000 buses pour former une nuée mouvante de cent cinquante-neuf jours.

Dans la même conférence, Nelly Wenger parle d'un rituel, d'une «fable historique et nationale». A Expo.02, ce rituel s'accomplit, s'acheva et s'abolit dans l'acte même de la performance. Dans les Expositions nationales, la représentation de la «fable» ne peut être permanente, et tire de son exceptionnalité même la possibilité d'une redéfinition de la nation et de son identité. Elle permet à l'Exposition actuelle de se démarquer des Expositions précédentes. La destruction volontaire d'Expo.02 est cependant unique par son ampleur, comme si, pour le pays, ce «rendez-vous avec lui-même», comme dit la présidente, se devait de rester sans lendemain.

\section{L'ARMÉE ET LES ÉGLISES}

«La Suisse aujourd'hui, démocratie modèle, prétend toujours être défendue par le peuple armé, chaque citoyen garde ses armes chez lui ${ }^{51}$, rappelle Dominique Schnapper, pour qui la citoyenneté implique la conscription. L'Armée et les Eglises sont, jusqu'à la seconde moitié du $\mathrm{XX}^{\mathrm{e}}$ siècle, des institutions consubstantielles d'une vision traditionnelle et largement partagée du caractère national

${ }^{51}$ D. Schnapper, La communauté des citoyens. Sur l'idée moderne de nation, op. cit. p. 109. 
aux Expositions nationales. «Les représentations de l'armée [...] font partie des domaines pour lesquels on dénote clairement la volonté d'amener les visiteurs à s'identifier à la Nation 'suisse' $»^{52}$, affirment Urs German et Agnes Nienhaus dans Expos.ch, même si ces institutions sont grevées, et l'Armée en premier lieu, de non-dits et d'une hostilité exprimés par certains secteurs de l'opinion, qui rendent leur représentation problématique. Au milieu du XIX $\mathrm{X}^{\mathrm{e}}$ siècle, l'Armée est une force unificatrice de l'Etat fédéral en formation et intégratrice des citoyens, au moment et au lendemain de la sécession des cantons catholiques (Sonderbund en 1847-48); mais dans le dernier tiers du siècle, elle réprime les mouvements de grève, entre autres, en 1875 , celui des travailleurs occupés à la construction du tunnel du Gothard dont l'achèvement allait être célébré à l'Exposition de Zurich en 1883. Les milieux pacifistes et la gauche considèrent l'armée avec méfiance, pour le moins. Pour eux, elle est étroitement liée aux couches conservatrices et aux possédants.

Deux des Expositions nationales ont eu lieu l'année du déclenchement des guerres mondiales, et celle de 1964 au lendemain de la Guerre froide. On comprend que la présentation de l'Armée y fut un enjeu considérable.

Les relations difficiles entre les cantons catholiques et l'Etat fédéral dominé par les cantons protestants au XIX ${ }^{\mathrm{e}}$ siècle sont pour l'essentiel occultées dans les Expositions nationales. La religion occupe une place fondamentale dans la vision qu'ont les élites des valeurs suisses; elle jouit d'un statut officiel dans la plupart des constitutions cantonales, et donc dans les rituels civiques. Dans les Expositions, la paix confessionnelle et le fait qu'elles ont toutes lieu dans les chefs-lieux des cantons majoritairement protestants amène, de 1883 à 1964, l'expression d'une religiosité plus déiste que confessionnelle, largement présente dans les cérémonies officielles. Cette religiosité fonde, pour la classe politique du centre, les «hautes valeurs suisses», telles la «neutralité charitable», la démocratie consensuelle, l'humanitarisme de la Croix-Rouge et, bien sûr, en 1939, la « défense spirituelle».

L'Armée tout d'abord. A Paris, lors de l'Exposition universelle de 1867, Victor Cosse met au crédit de la Suisse la médiocrité des armes qu'elle exhibe dans son pavillon. Rappelant la signature, à Genève en 1864, de la première Convention sur la protection des blessés de guerre, il écrit: «J'y vois le vrai caractère d'une nation républicaine. Inhabile, indifférente au moins, aux travaux de la guerre, elle réserve son activité, ses forces, son intelligence pour ces œuvres moins éclatante, mais plus fécondes qui préparent un avenir de paix et de progrès $»^{53}$.

Absente de Zurich en 1883, l'Armée a son pavillon consacré à l' «Art militaire » à Genève en 1896, grâce à l'appui du Département militaire fédéral. On y voyait la totalité ou presque des armes et munitions qu'un règlement fédéral venait d'unifier, selon les règles de l'exhaustivité didactique qui prévalaient dans ce type de manifestation.

Mais en 1914 à Berne, l'Armée est désormais présente comme emblème et instrument de la neutralité armée et de la cohésion nationale, par-dessus le fossé

${ }^{52}$ U. German, A. Nienhaus, «Landesaustellungen und Armee - vier Bildkommentare», in: Expos.ch. Idées, intérêts, irritations, op. cit., p. 68.

${ }^{53}$ V. Cosse, «Les Beaux-arts de Suisse», op. cit., p. 308. 
séparant les Romands des Alémaniques. On y voit l'embryon de l'aviation militaire suisse, ainsi que la production moderne d'engins explosifs, présentés d'une façon élégante comme des objets ménagers dans une foire commerciale.

L'Armée occupa une place éminente à l'Exposition de Zurich en 1939 dans l'affirmation de la volonté de défense, au sein d'une Europe qui entrait dans la guerre. Elle y apparut au carrefour de mythes et de valeurs où étaient exaltés le passé héroïque des Confédérés, la lutte, toujours défensive, contre des voisins plus puissants, et la défense des traditions démocratiques du pays. La notion de «défense spirituelle», ce supplément d'âme, associait en quelque sorte bras armé et principe religieux. Le mot d'ordre alliait la volonté, le devoir et le possible d'une résistance à la menace extérieure.

Les traits sous lesquels l'Exposition dépeignait la Suisse, qui allaient devenir négatifs dans l'Après-guerre: isolement, petitesse, prétention à la supériorité morale, ont alors un tour positif et servent de fondement à une doctrine alliant l'identité essentielle et l'attitude de circonstance fondées sur la perspective du danger. En 1939, l'Armée et son image, à cause de la menace réelle, relèguent alors à l'arrière-plan tout un folklore militaire ${ }^{54}$, présent dans les célébrations officielles et dans un associationnisme d'anciens conscrits (fêtes de tir, amicales...)

Dans les valeurs à défendre, fondements de l'entité nationale, entrent aussi, à côté de la démocratie directe, des vertus de la frugalité et de la piété, la diversité des origines, des langues et des croyances. «Ils ne sont pas taillés dans le même bois, mais le même esprit les anime», proclamait le Livre d'Or de l'Exposition nationale ${ }^{55}$, commentant une galerie de portraits représentant la diversité des types helvétiques. L'article d'Arthur Mojonnier, «Peuple et patrie ${ }^{56}$, dans le même ouvrage, fait allusion aux migrations diverses à l'origine de la population de la Suisse. Il donne pour la première fois, dans ce cadre, des statistiques précises des langues et confessions. La diversité devient ainsi un élément clé de l'identité nationale.

Le message de la Landi n'allait pas sans ambiguitté: l'affirmation de la volonté de défense s'accompagnait d'une certaine xénophobie, impliquant plusieurs omissions, entre autres celle des conflits sociaux, au nom de l'union patriotique.

Le thème de la neutralité armée et de la défense du pays survécut à la Seconde Guerre mondiale, à l'Exposition de 1964, mais sans la ferveur de Zurich. A Lausanne, il ne restait plus grand chose du «Sonderfall», ni de la «défense spirituelle ». Les responsables locaux de l'Exposition s'étaient opposés à ce que l'Armée ait un secteur particulier hors de la thématique générale, et souhaitaient la reléguer en dehors de l'espace de la manifestation, à l'autre bout de la ville. En vain. L'Armée suisse obtint son secteur équipé des moyens audiovisuels plus modernes, autour du thème de la "Suisse vigilante» et du hérisson, symbole de ses missions défensives, au crépuscule de la Guerre froide. Peur du communisme,

${ }^{54}$ Arnold van Gennep l'avait relevé lors de la Première Guerre mondiale déjà : «Folklore militaire suisse», Mercure de France, 16 mars, 1916, pp. 367-371.

55 E. Rimli et M. Pobé (rédacteurs), Le Livre d'Or de l'Exposition nationale 1939, Zurich, Verkehrsverlag S.A, 1940, p. 33.

56 A. Mojonnier, «Peuple et patrie», in, E. Rimli et M. Pobé (rédacteurs), Le Livre d'Or de l' Exposition nationale 1939, op. cit., pp. 12-90. 
crainte et désir d'une Europe en devenir, le contexte de 1964 était différent de celui de 1939, et l'adhésion était mitigée; on était loin de l'unanimisme saluant l'armée des citoyens et son rôle intégrateur.

A l'Expo.02 enfin, l'Armée en tant que telle fut absente de l'Exposition, même si elle finança le «Chantier naval» sur le site de Morat; on pouvait y voir, avançant sur le lac, un ponton présentant aux visiteurs la politique de sécurité de la Suisse: «gérer les nouveaux dangers ». L'Armée équipa un espace à elle en dehors du périmètre, mettant en avant son rôle dans la gestion des catastrophes et l'intervention humanitaire.

L'Armée, après 1939, cesse d'être au cœur des représentations identitaires de la Suisse et d'incarner la cohésion nationale; de 1964 à Expo.02, elle exprime plutôt un rapport malaisé et ambigu au monde, où entrent les thèmes contradictoires de la neutralité, de l'humanitaire, de la vigilance et de l'ouverture.

Dans le portrait que les Expositions proposent de la Suisse et des Suisses, ou dans le miroir qu'elles leur tendent, la religion incarnée par les Eglises protestante et catholique romaine occupent une place pas toujours facile à saisir. C'est en 1964 à Lausanne qu'elle est le plus clairement explicitée, sans doute parce que la dimension religieuse allait moins de soi que dans les expositions précédentes. La brochure trilingue intitulée Le christianisme et les Eglises à l'Exposition nationale s'ouvre par l'affirmation suivante: «Notre conception de l'homme et du citoyen est marquée essentiellement par le christianisme ${ }^{57}$; puis vient le rappel des origines chrétiennes de la Suisse: «le pays où nous vivons fut chrétien bien avant d'être un Etat indépendant ${ }^{58} »$; après le rappel de la croix qui marque le drapeau suisse, la déclaration commune des Eglises précise: «Sans Dieu et sans christianisme, l'histoire de ce pays perd son sens.» ${ }^{59} \mathrm{Il}$ n'était pas question alors des religions juive et musulmane.

En réalité, dans les Expositions précédentes, la présence du christianisme était donnée comme indissociable de l'identité helvétique, mais la neutralité confessionnelle avait conduit à une sorte de repli sur une présentation diffuse et implicite, ainsi que sur les aspects traditionnels et muséographiques des faits religieux. Il y a une église/temple dans les «Villages suisses » de Genève, Berne et Zurich; dans le premier, Crettaz et Michaelis-Germanier rappellent que la cloche de l'église fut dotée «d'un carillon capable de jouer à la fois de la manière catholique et de la manière protestante $»^{60}$. L'œcuménisme plus abouti de Lausanne, avec mobilier de culte prévu pour les différentes confessions et cérémonies communes, est dû au rapprochement interconfessionnel du temps, mais aussi à un affaiblissement des pratiques.

A Expo.02, les Eglises confièrent l'aménagement de sept pavillons à des artistes de divers pays, sur le site de Morat. Sous le titre général de «un ange passe», chacun d'eux représenta une «une dimension religieuse de l'existence», de l'origine du monde à la prière et aux mystères. Morat a donc rompu entièrement

\footnotetext{
${ }^{57}$ Le christianisme et les Eglises à l'Exposition nationale, 1964, p. 3.

${ }^{58}$ Ibid., p. 6.

59 Ibid.

${ }^{60}$ B. Crettaz, J. Michaelis-Germanier, Une Suisse miniature ou les grandeurs de la petitesse, op.
} cit., p. 97. 
avec la religiosité alpestre et le thème d'une Suisse bénéficiant d'une protection divine particulière. Individualisation, «deshelvétisation» allaient de pair avec une présentation des faits religieux hors du cadre confessionnel, dans laquelle l'affirmation de l'historicité chrétienne de la Suisse n'avait plus sa place.

\section{EXPO.02 : LA FIN DES EXPOSITIONS NATIONALES?}

Le projet d'une Exposition nationale pour 1991, sur le site historique de la Suisse primitive, avec un programme thématique sur les besoins fondamentaux, n'aboutit pas. Les budgets furent en effet refusés par les citoyens des cinq cantons concernés. On peut interpréter ce refus comme le rejet d'un projet d'experts, coûteux et imposé d'en haut à une population jugée à tort comme étant la gardienne des traditions helvétiques. Il traduit en fait une crise de la représentation de soi en Suisse, et l'ambiguité du rapport célébration-analyse-critique, déjà visible en 1964. Le modèle helvétique comme entité morale était lui aussi en crise. Le portrait était devenu flou, l'identité insaisissable. Un an après l'année prévue pour l'exposition désormais mort-née s'ouvrait celle, universelle, de Séville. Le pavillon helvétique s'intitulait précisément: La Suisse n' existe pas.

Les concepteurs de l'Expo.01, retardée en Expo.02, se devaient donc de rectifier les relations entre la future manifestation et le national. Pourtant le mandat resta, à peu de chose près, le même qu'au cours du siècle précédent. S'il ne fut plus question, comme en 1939, de «rappeler à notre peuple les fondements spirituels de la Confédération », et moins encore, comme en 1883, de donner une idée complète des forces productives de la Suisse, il s'agissait toujours de redéfinir et de représenter les «valeurs permanentes », d'ailleurs non précisées. La page web d'Expo.02 déclarait: «Comme toutes les nations nées d'une volonté commune, la Suisse 'Willensnation', doit périodiquement effectuer un retour sur elle-même pour se redéfinir et se fixer de nouveaux objectifs ${ }^{61}$, affirmant tout à la fois la nécessaire introspection et le retour de la nation. Alors que le Conseil fédéral donnait à Expo.02 le mandat de «manifester, par delà les frontières linguistiques, la cohésion de notre pays et sa confiance dans l'avenir au seuil d'un nouveau siècle $»^{62}$, le président de la Confédération, dans l'album officiel de l'Exposition, développait lui aussi le thème de la nécessaire cohésion: «Nous sommes un peuple qui rassemble plusieurs cultures et groupes linguistiques. Cette diversité fait la richesse particulière de notre Nation. Mais la cohésion d'un peuple aussi hétérogène n'est jamais assurée durablement. Il faut sans cesse y travailler.» ${ }^{63}$

Le recours à la notion de nation accompagne donc cet appel renouvelé à l'unité et à la cohésion, parallèlement au constat du pluralisme culturel et linguistique comme composante fondamentale de l'ensemble national. Il intervient dans un contexte difficile pour la cohésion souhaitée, dans lequel le principe même d'une Exposition est contesté, alors que s'accroissent dans l'opinion les

${ }^{61}$ Cité par F. Sardet: «Organiser l'Expo 64: espace, argent et pouvoirs », op. cit., p. 234.

${ }^{62}$ Forum helveticum, «Expo. 02 et cohésion nationale», op. cit., p. 6.

${ }^{63} \mathrm{~K}$. Villiger, «La Suisse veut évoluer avec ses valeurs traditionnelles », in ImagiNation. Le Livre officiel d'Expo.02, Lausanne, Payot, 2002, p. 7. 
divergences face un choix fondamental de l'adhésion à l'Europe, divergence quasi occultée dans l'Exposition.

Ses concepteurs ont renoncé à toute thématique traditionnelle helvétique, aux portraits sociologiques, à tout ce qui pouvait ressembler à la «Voie suisse », façon Lausanne 64 ou au «chemin des hauteurs », façon Zurich 1939, à toute évocation consensuelle d'une histoire, de valeurs communes.

Le choix d'une thématique délocalisée jouant sur des couples antithétiques: «Moi et l'Univers», « Nature et Artifice», «Pouvoir et Liberté»..., utilisant les ressources de l'art vidéo et les voies de l'hédonisme et du ludique, dont l'esthétique tenait davantage du «choc visuel» que de la présentation didactique, marquait une rupture avec le style d'exposition en usage jusqu'en 1964.

Les sites-événements étaient à première vue aux antipodes des consignes du mandat et de son message rassembleur. A y regarder de plus près, la thématique nationale n'était pas absente, il y avait un pavillon sur la politique de sécurité, un autre sur la Heimatfabrik (fabrique de la patrie), un autre encore sur les noms des communes suisses, mais le langage était celui des spécialistes des arts visuels.

Pour parler du traitement thématique selon cette approche particulière, qui selon lui réhabilite la catégorie du «national» en la rendant plus familière et plus proche des sens, le directeur artistique Martin Heller ${ }^{64}$ parle de «patriotisme light», expression qu'il a forgée avec le sociologue zurichois Kurt Imhof (2003: 10 ), fait de distance, d'ironie et d'affection ${ }^{65}$. «Le public suisse a découvert dans son Exposition nationale qu'une espiègle distance par rapport à lui-même rend davantage justice aux choses que la familière opiniâtreté avec laquelle une vieille frange gauchisante exerce systématiquement la critique ou, à l'opposé, l'Etat défend un patriotisme de bon aloi.» ${ }^{66}$

Les anciennes figures du folklore suisse, avec ses costumes et ses yodleurs, les restes de Festspiel y furent recyclés dans des spectacles où se mêlaient citations anciennes et frissons de l'avant-garde.

L'Expo.02 a eu l'ambition d'offrir un cadre passager, hors de l'ordinaire, à une nouvelle expression du «national», comme lieu de création et de rencontre. Genre d'un autre siècle, l'Exposition nationale n'a pu rendre explicite ni le pluralisme culturel, ni l'exigence de cohésion nationale. Elle fit figure d'une vaste surpriseparty, d'une fête sympathique et diffuse. «Toute la Suisse a eu du plaisir, et ce plaisir n'avait pas d'identité liée à une région linguistique en particulier», conclut la table ronde sur la cohésion nationale réunie à l'issue de l'Exposition ${ }^{67}$.

Le portrait de la Suisse est désormais introuvable ${ }^{68}$. Lorsqu'on a renoncé à la représenter, à chercher son «contenu », que ce soit en termes de propriétés sociologiques, morales, caractérielles, il reste la possibilité de parler de la Suisse en terme de Nation.

${ }^{64}$ Je remercie vivement Martin Heller pour l'entretien qu'il m'a accordé sur Expo.02 en juin 2004. Bien entendu, je garde l'entière responsabilité des lignes qui s'y rapportent.

${ }^{65}$ M. Heller, «Zur Erfahrung des Nationalen: Bericht eines Novizen », Forum helveticum, Lenzburg, 2003, p. 10.

${ }^{66}$ ImagiNation, Le Livre officiel d'Expo.02, Lausanne, Payot, 2002, p. 291.

${ }^{67}$ Forum helveticum, «Expo. 02 et cohésion nationale», op. cit., p. 35.

${ }^{68}$ P. Centlivres, «Le portrait introuvable: la Suisse des expositions nationales », op . cit. 